\title{
Optimisation du comportement thermique du transistor à haute mobilité d'électron (HEMT) par la méthode KA-CMA-ES
}

\section{Thermal behavior optimization of the high electron mobility transistor}

\author{
(HEMT) using the KA-CMA-ES method
}

\author{
Amar Abdelhamid ${ }^{1}$, Radi Bouchaïb ${ }^{2}$, El Hami Abdelkhalak ${ }^{3}$ \\ ${ }^{1}$ Laboratoire LIMII, FST, Hassan First University of Settat, Maroc, amarabdelhamid20@gmail.com. \\ ${ }^{2}$ Laboratoire LIMII, FST, Hassan First University of Settat, Maroc, bouchaib.radi@yahoo.fr. \\ ${ }^{3}$ Laboratoire LMN, INSA, Rouen, France, Abdelkhalak.elhami@insa-rouen.fr.
}

RÉSUMÉ. L'objectif principale de L'optimisation est d'assurer une conception robuste des systèmes avec un cout minimal, dans ce papier on s'intéresse à l'optimisation du comportement du transistor à haute mobilité d'électrons (HEMT), il est un élément très important dans les systèmes mécatroniques à haute puissance. II est composé de plusieurs couches de matériaux, les paramètres géométriques et thermiques de ces couches influencent le comportement thermique et notamment la température de fonctionnement du transistor, d'où sur sa performance. La méthode CMA-ES assistée par le krigeage (KA_CMA_ES) codée sur Matlab couplé avec un modèle éléments finis développé sur Comsol multiphysics, ce couplage a permis d'optimiser la structure de transistor afin de réduire sa température maximale du fonctionnement, pour que le transistor assure sa fonction avec moins d'influence sur les autres caractéristiques. Une comparaison entre la méthode KACMAES et CMA-ES a été faite. La méthode KA-CMAES a montré une efficacité en termes de précision et de temps du calcul.

ABSTRACT. The main objective of Optimization is to ensure a robust system design with minimal cost, in this paper we focus on the optimization of the behavior of the High Electron Mobility Transistor (HEMT), it is a very important element in high power mechatronic systems. It is composed of several layers of materials, the geometrical and thermal parameters of these layers influence the thermal behavior and in particular the operating temperature of the transistor, hence its performance. The CMA-ES method assisted by kriging (KA_CMA_ES) coded on Matlab coupled with a finite element model developed on Comsol multiphysics, this coupling allowed to optimize the transistor structure in order to reduce its maximum operating temperature, so that the transistor performs its function with less influence on the other characteristics. A comparison between the KA-CMA-ES and CMA-ES methods was made. The KA-CMA-ES method showed an efficiency in terms of accuracy and computation time.

MOTS-CLÉS. Optimisation, HEMT, KA-CMA-ES, systèmes mécatroniques.

KEYWORDS. Optimization, HEMT, KA-CMA-ES, Mecatronics systems.

\section{Introduction}

Au sien des systèmes complexes, le transistor est un élément très importent, il assure plusieurs fonctions comme la commande, l'amplification et la minimisation des signaux. Parmi ces transistors on cite le transistor à haute mobilité d'électron (HEMT) à base de nitrure d'aluminiumgallium/nitruregallium $(\mathrm{AlGaN} / \mathrm{GaN})$, c'est un composant électronique très puissant grâce à sa structure et ses matériaux [1]. Ce transistor a été utilisé dans plusieurs applications à haute fréquence et à haute température[2] [GAR 16], tels que la télécommunication, la guerre électronique et les systèmes aéroportés [3]. Le HEMT a été utilisé aussi dans plusieurs systèmes tels que les amplificateurs à haute puissance, les radars et les satellites, il se trouve aussi dans les capteurs et les dispositifs à radiofréquence [4]. En plus, la température de fonctionnement est un facteur très important, elle influence la fiabilité du HEMT, car la plupart de ses caractéristiques telle que la mobilité des électrons, la conductivité thermique, la vitesse de saturation et autres sont en fonction de la température [5]. Toutes ces caractéristiques ont tendance à se dégrader avec l'élévation de la 
température à cause de phénomène de l'auto-échauffement du transistor [6]. Par conséquent ça donne naissance à des dégradations comme l'enterrement de la grille, endommagement de la connexion entre la puce et le boitier, la dégradation de la mobilité des électrons et la réduction de courant [7]. Dans le cas des systèmes aéroportés, la majorité des défaillances sont causés par des amplificateurs à haute puissance au niveau de ses transistors à haute puissance, ces défaillances sont à cause de la température de fonctionnement des transistors qui influence négativement leur fiabilité et donc celle de système [8].

Pour assurer le bon fonctionnement du HEMT, il est nécessaire d'améliorer son comportement thermique, pour que sa température ne dépasse pas des valeurs critiques. Par conséquent, il faut penser à diminuer la température maximale du fonctionnement du HEMT D'autre part, l'optimisation de dimensionnement sert à trouver les paramètres de la conception qui minimise une fonction objective. Dans ce sens CMA-ES (Covariance Matrix Adaptation-Evolution Strategy) est une méthode de recherche stochastique basée sur la population dans des espaces continus et discrets [9], elle sert à résoudre des problèmes d'optimisation par la minimisation d'une fonction objective, la fonction objective à minimiser est la température maximale de fonctionnement du transistor. Cette méthode a montré une efficacité à travers des résultats obtenus dans un travail que nous avons déjà fait qui vise l'optimisation de comportement thermique du HEMT [10]. Mais cette méthode reste couteuse et impraticable en terme du temps, dans le cas où on aura besoins d'effectuer plusieurs(milles) simulation ou on a un modèle éléments finis qui nécessite un temps de calcul important. Pour dépasser cette contrainte, dans ce travail on vise a réalisé une optimisation du comportement thermique en utilisant la méthode CMA-ES assistée par le Krigeage (KA-CAM-ES).

Dans un premier temps, on va faire une description du HEMT : structure, principe du fonctionnement, avantages et utilisations. Par la suite on va développer un modèle thermique permettant de simuler le comportement thermique du HEMT et identifier les paramètres à minimiser. Ensuite on définit la méthode KA-CMA-ES et ses paramètres. Dans la partie suivante, un couplage entre les deux logiciels Matlab et Comsol multiphysics sera développé, il permet de connecter les deux modèles thermique et statistique afin de résoudre le problème de l'optimisation. A la fin on extrait les résultats et on fera une comparaison de la variation de la température de fonctionnement du HEMT avant et après l'optimisation. Et une comparaison entre les résultats obtenus par la méthode CMA-ES et la méthode KA-CMA-ES.

\section{Description du composant}

Le HEMT possède une structure très spéciale, il est composé de plusieurs couches de différents matériaux, souvent il est construit sur un substrat en $\mathrm{SiC}$ (carbure de silicium), puis une couche de nucléation séparant le substrat et la couche de $\mathrm{GaN}$, une couche en $\mathrm{AlGaN}$ sert à créer une hétérojonction avec la couche de GaN pour produire le gaz 2DEG qui représente le canal des électrons et autres couches qui assures autre fonctions [11] [12]. Après une étude bibliographique, on a constaté que le HEMT a plusieurs structures, et qu'il y a une différence entre ces structures concernant la topologie et les matériaux utilisé [13] [14] [15] [16], tout ça nous ramène à conclure que le transistor à haute mobilité est une technologie qui est encore de développement et d'amélioration, afin de trouver la meilleure structure de HEMT qui permet d'augmenter la performance et sa fiabilité. Pour réaliser notre étude la structure utilisé est montrée dans la figure 1.1 , on a utilisé comme données géométriques la longueur de la source et du drain $\mathrm{Ls}=\mathrm{Lg}=45 \mu \mathrm{m}$, la longueur de grille $\mathrm{LG}=1 \mu \mathrm{m}$, la largeur de grille $100 \mu \mathrm{m}$. Pour les couches on a l'épaisseur du substrat 100 $\mu \mathrm{m}$, épaisseur de Gan $3 \mu \mathrm{m}$ et AlGaN $0.3 \mu \mathrm{m}$. 


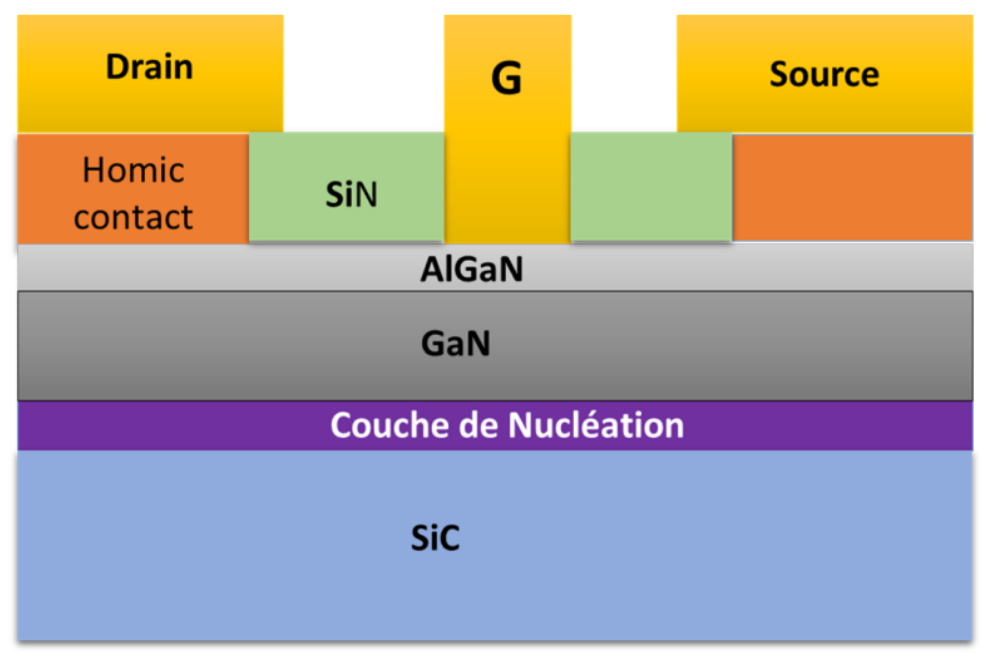

Figure 1. Evolution de la température en fonction de la puissance dissipée.

\section{Modèle éléments finis}

Lors de son fonctionnement, Le HEMT dissipe une puissance (Pdiss) au niveau de la zone active par le phénomène de l'auto-échauffement, cette puissance est en fonction de la tension (Vds) et le courant (Ids) qui circule entre le drain et la source, elle a comme expression [17]:

$$
\mathrm{P}_{\mathrm{diss}}=\mathrm{V}_{\mathrm{ds}} * \mathrm{I}_{\mathrm{ds}}
$$

Afin d'étudier l'effet de la puissance dissipe sur le comportement thermique du HEMT, une modélisation thermique est nécessaire, cependant, il faut étudier le transfert thermique au sein du transistor, le mode du transfert principal est la conduction et l'équation de chaleur sera [18] :

$$
k \cdot \nabla^{2} T=\rho \cdot C_{P} \cdot \frac{\partial T}{\partial t}+Q
$$

avec:

-Q puissance dissipée en joules;

- K conductivité thermique $(\mathrm{w} / \mathrm{m} / \mathrm{k})$;

- $\rho$ densité Kg.m ${ }^{-3}$;

- Cp capacité massique thermique $\left(\mathrm{J} \cdot \mathrm{Kg}^{-1} \cdot \mathrm{k}^{-1}\right)$;

- T température $(\mathrm{K})$

Cette modélisation sera mise en place en utilisant la méthode des éléments finis, elle permet de résoudre l'équation de chaleur et d'observer l'influence des paramètres thermique et géométrique sur le comportement thermique du HEMT. Les propriétés des matériaux utilisés dans la modélisation : la conductivité thermique, le densité volumique, la capacité thermique massique avec leurs valeurs, sont mentionnées dans la tableau 1 [8], [19]. Les propriétés de ces matériaux ainsi que les caractéristiques du HEMT sont en fonction de la température du fonctionnement. La conductivité thermique, la mobilité des élections, la vitesse de saturation et autres propriétés ont tendance à se dégrader avec l'augmentation de la température du fonctionnement du transistor, 
cette température peut atteindre des valeurs critiques à cause de phénomène de l'autoéchauffement[6], [20]. On constate que l'élévation de la température influence la fiabilité et la performance du HEMT, d'où la nécessité de chercher à diminuer cette température pour augmenter la fiabilité. Pour réaliser ce but, des méthodes seront utilisés comme la méthode des éléments finis et la méthode CMA-ES.

\begin{tabular}{|c|c|c|c|}
\hline Matériaux & $\rho[\mathrm{kg} / \mathrm{m} 3]$ & $\mathrm{KTH}[\mathrm{W} / \mathrm{m} / \mathrm{K}]$ & $\mathrm{Cp}[\mathrm{J} / \mathrm{kg} / \mathrm{K}]$ \\
\hline Au & 19300 & 310 & 137 \\
\hline SiN & 3300 & 10 & 713 \\
\hline AlGaN & 5470 & $\left.25 \cdot \frac{293}{273+T}\right)^{1,35}$ & 548 \\
\hline GaN & 6100 & $\left.161 \cdot \frac{293}{273+T}\right)^{1,45}$ & 490 \\
\hline SiC & 3220 & $\left.416 \cdot \frac{293}{273+T}\right)^{1,5}$ & 690 \\
\hline AlN & 6100 & $\left.6,7 \cdot \quad \frac{293}{273+T}\right)^{2,7}$ & 490 \\
\hline
\end{tabular}

Tableau 1. Propriétés des matériaux.

Le modèle éléments fins est développé sur le logiciel éléments finis Comsol multiphyscs, il permet d'étudier l'influence de plusieurs paramètres du HEMT sur sa température du fonctionnement, la figure 2.2 montre l'influence de la puissance dissipée (représentant l'un des conditions du fonctionnement) sur la variation de la température de HEMT[11].

La modélisation thermique du transistor par la méthode des éléments finis (MEF) a permis d'identifier les paramètres de la géométrie, qui ont un grand impact sur la température de HEMT. L'épaisseur du substrat (a), l'épaisseur de la couche de nucléation (b) et le développement du transistor (c), sont les trois paramètres (en $\mu \mathrm{m}$ ) qui ont une grande influence sur le comportement thermique du transistor. Ce modèle éléments finis sera Le but de l'optimisation dans ce cas est de minimiser la température maximale du fonctionnement du transistor.

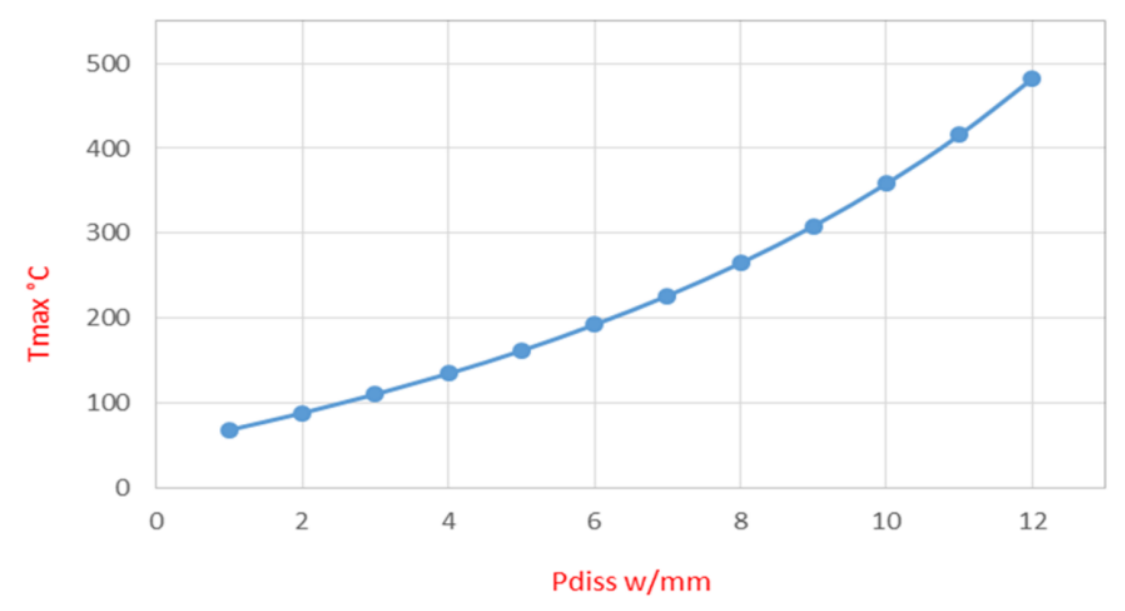

Figure 2. Evolution de la température en fonction de la puissance dissipée 


\section{Covariance Matrix Adaptation-Evolution Strategy ( CMA-ES )}

La méthode CMA-ES(Covariance Matrix Adaptation-Evolution Strategy) est le meilleur membre de la famille des algorithmes d'évolution, est une méthode d'optimisation stochastique sans dérivée et peut s'adapter à des problèmes d'optimisation non convexe, CMA-ES est algorithme d'optimisation plus puissant par rapport aux autres, elle est basée sur les quatre principes de la sélection naturelle : évolution, sélection, recombinaison et mutation [9]. La recombinaison des éléments (parents) d'une population génère de nouveaux individus (enfants), les nouveaux individus subissent une mutation pour qu'ils deviennent des nouveaux membres de la même population, afin de garder et adapter la taille de population une sélection est faite en choisissant les meilleurs individus après la mutation. La mise en place des opérations évolutionnaires assure cette démarche, pour guider la démarche vers un optimum globale dans un espace de recherche continu ou discret [21]. CMA-ES $(\lambda, \mu)$ est basé sur l'adaptation de la matrice de covariance de la loi multi normale dans $R^{n}$, cette adaptation est équivalent de la construction d'une approximation de la fonction objectif $\mathrm{f}$, cette méthode génère 1 nouveaux individu a partir de mu éléments de la population. CMA-ES $(\lambda, \mu)$ génère la population en échantillonnant une distribution multi normale [22]:

$$
\boldsymbol{x}_{k}^{(g+1)} \sim \boldsymbol{m}^{(g)}+\sigma^{(g)} \mathcal{N}\left(\mathbf{0}, \boldsymbol{C}^{(g)}\right) \quad \text { fork }=1, \ldots, \lambda
$$

avec :

indique la même répartition à gauche et à droite.

$\mathcal{N}\left(\mathbf{0}, \boldsymbol{C}^{(g)}\right)$ est une distribution normale multivariée avec une moyenne nulle et une matrice de covariance $\mathrm{C}(\mathrm{g})$.

$\mathrm{X}^{(g+1)} \in \mathbb{R}^{n}, k$ ième progéniture (individu, point de recherche) de la génération $\mathrm{g}+1$.

$\mathrm{m}(\mathrm{g}) \in \mathrm{Rn}$, valeur moyenne de la distribution de la recherche à la génération $\mathrm{g}$.

$\sigma(\mathrm{g}) \in \mathrm{R}>0$, la taille de pas, à la génération $\mathrm{g}$.

$\mathrm{C}(\mathrm{g}) \in \mathrm{Rn} \times \mathrm{n}$, matrice covariance à la

génération $\mathrm{g}$.

$\lambda \geq 2$, la taille de la population.

Par suite, les images des individus générés par échantillonnage sont évaluées par la fonction objectif, les meilleurs individus seront regroupés dans le vecteur moyen $\mathrm{m}$, il a comme expression :

$$
\boldsymbol{m}^{(g+1)}=\sum_{i=1}^{\mu} w_{i} \boldsymbol{x}_{i: \lambda}^{(g+1)}
$$

et

$$
\sum_{i=1}^{\mu} w_{i}=1, \quad w_{1} \geq w_{2} \geq \cdots \geq w_{\mu}>0
$$

avec :

$\mu \leq \lambda$ le nombre des points sélectionnés ou la taille de la population originale (parents).

wi $=1 \ldots \mu \in \mathrm{R}>0$, représente les pois de recombinaison, sont strictement positifs et vérifiant l'équation [5]. xi: $\lambda$ : le iene meilleur individu sélectionné de l'ensemble des individus $[\mathrm{x} 1, \mathrm{x} 2, \cdots, \mathrm{x} \lambda]$ générés par l'équation [3]. 
i : $\lambda$ est l'indice du ieme individu après classement tel que, $f(x 1: \lambda) \leq f(x 2 \cdot \lambda) \leq \cdots \leq f(x \lambda: \lambda)$, où $f$ est la fonction objectif.

Après la mise à jour du vecteur moyen $\mathrm{m}$, l'étape suivante est l'adaptation de pas $\sigma$ et la matrice de covariance C.la mise à jour des deux paramètres se fait par deux chemins d'évolution pc et $\mathrm{p}_{\sigma}$ [24]. Cependant, la suite du processus de la méthode CMA-ES nécessite d'actualiser le chemin d'évolution $\mathrm{p}_{\sigma}$ permettant de mettre à jour la taille du pas $\sigma$, les deux paramètres se calculent suivant les équations suivantes :

$$
\begin{aligned}
& \mathbf{p}_{\sigma}^{(g+1)}=\left(1-c_{\sigma}\right) \mathbf{p}_{\sigma}^{(g)}+\sqrt{c_{\sigma}\left(2-c_{\sigma}\right)} \sqrt{\mu_{w}}\left(\mathbf{C}^{(t)}\right)^{-\frac{1}{2}} \frac{\mathbf{m}^{(g+1)}-\mathbf{m}^{(g)}}{\sigma^{(t)}} \\
&=\left(1-c_{\sigma}\right) \mathbf{p}_{\sigma}^{(g)}+\sqrt{c_{\sigma}\left(2-c_{\sigma}\right)} \sqrt{\mu_{w}} \sum_{i=1}^{\mu} w_{i} \mathbf{z}_{i \lambda} \\
& \sigma^{(g+1)}=\sigma^{(g)} \exp \left(\frac{c_{\sigma}}{d_{\sigma}}\left(\frac{\left\|\mathbf{p}_{\sigma}^{(g+1)}\right\|}{\mathbb{E}\left(\left\|\mathcal{N}_{d}(\mathbf{0}, \mathbf{I})\right\|\right)}-1\right)\right.
\end{aligned}
$$

$p_{\sigma}^{(g)} \in \mathbb{R}^{d} \quad:$ le chemin d'évolution pour la taille du pas à la génération $\mathrm{g}$.

$\mathrm{c}_{\sigma} \in[0,1]$ : la constante du temps pour l'adaptation de la taille du pas.

$\mu_{\mathrm{w}}$ : appelé variance masse de sélection effective (variance effective sélection mass).

Pour mettre à jour la matrice de covariance $\mathrm{C}$, il faut actualiser le chemin d'évolution pc, ça se fait suivant les équations suivantes :

$$
\begin{aligned}
\mathbf{p}_{c}^{(g+1)} & =\left(1-c_{c}\right) \mathbf{p}_{c}^{(g)}+h_{\sigma} \sqrt{c_{c}\left(2-c_{c}\right)} \sqrt{\mu_{u}} \frac{\mathbf{m}^{(g+1)}-\mathbf{m}^{(g)}}{\sigma^{(g)}} \\
& =\left(1-c_{c}\right) \mathbf{p}_{c}^{(g)}+h_{\sigma} \sqrt{c_{c}\left(2-c_{c}\right)} \sqrt{\mu_{w}} \sum_{i=1}^{\mu} w_{i}\left(\mathbf{C}^{(g)}\right)^{\frac{1}{2}} \mathbf{z}_{i: \lambda}
\end{aligned}
$$

Alors que la matrice de covariance devient comme suit :

$$
\begin{aligned}
C^{(g+1)}= & \left(1-c_{1}-c_{u}\right) \mathbf{C}^{(g)}+c_{1}\left(\mathbf{p}_{c}^{(g+1)}\left(\mathbf{p}_{c}^{(g+1)}\right)^{\mathrm{T}}+\delta\left(h_{\sigma}\right) \mathbf{C}^{(g)}\right) \\
& +c_{\mu} \sum_{i=1}^{u} w_{i}\left(\left(\mathbf{C}^{(g)}\right)^{\frac{1}{2}} \mathbf{z}_{i, \lambda}\right)\left(\left(\mathbf{C}^{(g)}\right)^{\frac{1}{2}} \mathbf{z}_{i: \lambda}\right)^{T}
\end{aligned}
$$

Avec : $\mathrm{p}_{\mathrm{c}}{ }^{(\mathrm{g})} \in \mathrm{R}^{\mathrm{d}}$ : le chemin d'évolution pour la taille du pas à la génération $\mathrm{g}$, sa valeur initiale est $\operatorname{pc}(0)=0 \mathrm{cc} \in[0,1]$ : la constante d'adaptation de la matrice de covariance. $\mathrm{h}_{\sigma}$ : est la fonction d'Heaviside. $c_{1}$ et $c_{\mu}$ : exprime le taux d'apprentissage (learning rate) respectivement pour rank-oneupdate et rank- $\mu$-update $\left.\mathrm{C}^{(t)}\right)^{\frac{1}{2}}$, leurs valeurs par défaut sont $c_{1}=\frac{2}{(d+1.3)^{2}+\mu_{u v}}$, et $c_{u}=\min \left(1-c_{1}, 2 \frac{\mu_{v}-2+1 / \mu_{w}}{(d+2)^{2}+\alpha_{u} \mu_{w} / 2}\right)$

avec $\alpha \mu=2$.

$\delta\left(h_{\sigma}\right)=\left(1-h_{\sigma}\right) c_{c}\left(2-c_{c}\right)$. Toutes ces étapes peuvent s'organiser dans un algorithme, qui s'exécute en fonction des conditions de convergence choisis, suivant un ordre bien précis, l'algorithme se répète jusqu'à la satisfaction de la condition de convergence en retournant le meilleur individu. 
Toutes les étapes nécessaires de la méthode CMA-ES sont ordonnées suivant un algorithme bien détaillé dans [23] [24].

\section{Métamodèle de krigeage}

Afin de dépasser la contrainte du temps du calcul dans le processus de l'optimisation, nous avons développé une nouvelle approche de l'optimisation, cette approche est basée sur les métamodèles. Les métamodèles sont des modèles simplifiés des modèles de simulation détaillés. Les évaluations à l'aide de métamodèles sont plus efficaces et plus praticables par rapport aux modèles détaillés en termes du temps. Parmi les types des métamodèles les plus utilisé, on trouve le métamodèle de krigeage. Le modèle de krigeage est une modélisation basée sur un processus gaussien pour interpoler des données déterministes sans bruit. Le modèle de krigeage est donné sous la forme [25]:

$$
Y(x)=m(x)+Z(x)
$$

Cette forme contient deux composantes principales : $\mathrm{m}(\mathrm{x})$ est une fonction déterministe ou fonction de tendance globale, et la fonction aléatoire gaussienne $\mathrm{Z}(\mathrm{x})$ qui est un processus gaussien stationnaire, c'est-à-dire de moyenne constante. On le suppose souvent centré : $E(Z(x))=0 \forall x$ et dont la covariance entre deux points ne dépend que de leur distance.

\section{La méthode CMA_ES assistée par le krigeage.}

La méthode CMA-ES est une approche efficace qui permet de résoudre des problèmes de l'optimisation, la seule contrainte apparait dans le temps de calcul nécessaire pour trouver le meilleur optimum, car CMA-ES est base sur le modèle éléments finis qui nécessite parfois des dizaines de minutes, ce qui rend cette approche très couteuse en terme du temp du calcul. Pour rendre CMA-ES plus efficace on a développé un couplage le modèle de krigeage et la méthode de CMA-ES, ce couplage va permettre au d'évaluer la fonction objectif à partir du métamodèle sans avoir besoin d'appeler le modèle éléments finis à chaque itération. Le couplage entre la méthode CMA-ES et le métamodèle de krigeage donne naissance à une nouvelle approche qu'on appellera CMA-ES assisté par le krigeage (KA-CMA-ES), cette approche s'exécute suivant un algorithme bien précis [21][26].

\section{Algorithme 1 : CMA-ES assistée par le krigeage.}

Initialiser les paramètres de CMA-ES $C(0)=I, \sigma^{(0)}$ et $p_{s}^{(0)}=0, p_{c}^{(0)}=0$,

Initialiser le vecteur moyen $m^{(0)}$; préciser les conditions de convergence ;

Le nombre de génération $g \leftarrow-0$

Tant que " la condition de convergence n'est pas vérifiée "

$\left.x g_{i}+1 \sim \mathbf{m}^{(g)}+\sigma^{(g)} \mathcal{N} 0, C^{(g)}\right)$, for $i=1, \ldots, \lambda$

Faire : Générer l'ensemble d'entrainement $\mathrm{T}$ :

$T \leftarrow\left\{\left(x_{i}, f_{i}\right) \in \mathcal{S} \|\left(x_{i}-m^{(b)}\right)^{T}\left((\infty)^{2} c^{4}\right)-1\left(x_{i}-m^{(y)}\right) \leq r^{2}\right\}$

Si $|T|<n_{k}$ donc

Evaluer la fonction coût à partir de MEF.

Sinon : Evaluer la fonction coût à partir de métamodèle. 
Classer les meilleurs individus générés

Déterminer le vecteur moyen par mutation des $\mu$ meilleurs individus selon : $\mathbf{m}^{(g+1)}=\sum_{i=1}^{\mu} \omega_{i} z_{i}^{(g+1)}$

Mettre à jour le chemin d'évolution $P_{(\sigma)}$ Mettre à jour la taille de pas $\sigma^{(g+1)}$

Mettre à jour le chemin d'évolution $P_{(c)}$

$$
p_{c}^{(g+1)}=\left(1-c_{c}\right) p_{c}^{(g)}+h_{\sigma} \sqrt{c_{c}\left(2-c_{c}\right) \mu_{e f f}} \frac{m^{(g+1)}-m^{(g)}}{\sigma^{(g)}}
$$

Mettre à jour la matrice de covariance $C^{(g+1)}$

$$
C^{(g+1)}=\left(1-c_{1}-c_{u}+c_{1} \delta\left(h_{\sigma}\right)\right) C^{(g)}+c_{1} p_{c}^{(g+1)}\left(p_{c}^{(g+1)}\right)^{T}+c_{\mu} \sum_{i=1}^{u} w_{i}\left(\frac{x_{i: \lambda}^{(g+1)}-m^{(g)}}{\sigma^{(g)}}\right)\left(\frac{x_{i=1}^{(g+1)}-m^{(g)}}{\sigma^{(g)}}\right)^{T}
$$

\section{Fin tant que}

Retourner les meilleurs individus $x_{i}{ }_{i}+1$

\section{Problème de l'optimisation}

Le modèle éléments finis développée sous Comsol multiphysics a permis de simuler le comportement thermique du transistor, en plus il permet d'identifier les paramètres géométrique représentant un grand impact sur la température du fonctionnement du HEMT. L'épaisseur du substrat (a), l'épaisseur de la couche de nucléation (b) et le développement (c), sont les trois paramètres (en $\mu \mathrm{m}$ ) qui ont un grand impact sur le comportement thermique du transistor. Le but est de minimiser la température de fonctionnement du HEMT en agissant sur ces paramètres, par suite la fonction objectif sera la température maximale du fonctionnement. Le problème d'optimisation est :

$$
\left\{\begin{array}{c}
\min (f(x))=\min \left(\mathrm{T}_{\max }\right) \\
90<a<150 \\
0.1<\mathrm{b}<1 \\
80<\mathrm{c}<140
\end{array}\right.
$$

Afin de résoudre ce problème d'optimisation, un coulage des deux modèles : thermique et statistique sera développé. Le modèle thermique est représenté par le modèle éléments finis développé sous le logiciel Comsol multiphisics, le modèle statistique est représenté par la méthode KA-CMA-ES codée sous le logiciel Matlab. L'intégration des deux modèles se fait en respectant les instructions de l'algorithme 1. L'exécution de l'organigramme de couplage se fait par plusieurs étapes, elle commence par l'initialisation des paramètres d'optimisation dans le code Matlab, les étapes suivants seront suivant l'algorithme 1, le calcul des images des individus s'effectue soit en appelant le modèle éléments finis sous le logiciel Comsol soit par le modèle de krigeage sous Matlab. Quand le critère de l'arrêt cité dans l'initialisation est satisfait on obtient les valeurs optimums des paramètres choisis. 


\section{Résultats numériques}

Pour résoudre le problème de l'optimisation de ce travail, on a exécuté le processus de l'optimisation développé précédemment, les résultats obtenus sont mentionnés dans le tableau 7.2 : ce tableau contient les valeurs initiales et les valeurs optimales des paramètres choisis obtenues par les deux méthodes CMA-ES et KA-CMA-ES.

\begin{tabular}{|c|c|c|c|}
\hline Paramètres $(\mu \mathrm{m})$ & Valeurs initiales & $\begin{array}{c}\text { Valeurs optimisées } \\
\text { KA-CMA-ES }\end{array}$ & $\begin{array}{c}\text { Valeurs optimisées } \\
\text { CMA-ES }\end{array}$ \\
\hline $\mathrm{a}$ & 100 & 98.21 & 97.33 \\
$\mathrm{~b}$ & 0.8 & 0.381 & 0.353 \\
\hline $\mathrm{c}$ & 100 & 118.60 & 119.13 \\
\hline Temps $(\mathrm{s})$ & --- & 31450 & 39553 \\
\hline
\end{tabular}

Tableau 2. Les paramètres de la géométrie du HEMT à optimiser

Le tableau suivant représente les valeurs initiales avant l'optimisation et les valeurs optimums après l'optimisation, on constate que les deux méthodes permettent de résoudre le problème de l'optimisation traité, mais on remarque bien que la méthode KA-CMA-ES est plus efficace en terme du temps du calcul. Alors que CMA-ES reste couteuse.

Pour mesurer l'influence de ces résultats sur le comportement thermique du transistor. On construit un nouveau modèle avec les paramètres géométriques optimums, des simulations numériques ont été effectuées en gardant les mêmes conditions de fonctionnement (conditions aux limites) et les autres paramètres constants. Afin d'observer l'influence des valeurs optimums sur l'évolution de la température dans les différentes valeurs de la puissance dissipée dans le HEMT, la figure 4 représente l'évolution de la température en fonction de la puissance dissipée dans les deux cas : avant et après l'optimisation par les deux méthodes, il est clair aussi que l'évolution de la température est moins importante après l'optimisation.

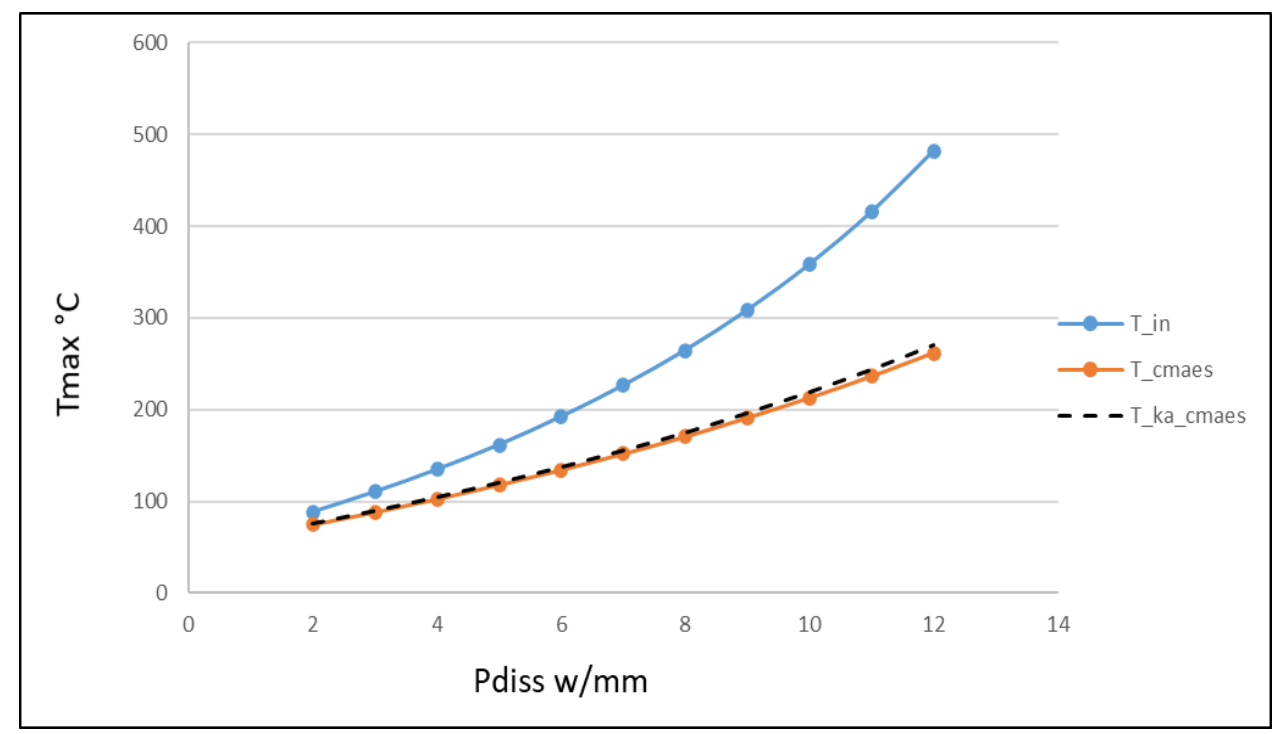

Figure 3. Evolution de la température du HEMT avant et après l'optimisation 
D'après la figure 4 on remarque qu'avant d'optimiser la structure du transistor sa température maximale du fonctionnement peut atteindre plus de $490{ }^{\circ} \mathrm{C}$, sous une puissance dissipée de 12 $\mathrm{w} / \mathrm{mm}$ et une température de référence de $50^{\circ} \mathrm{C}$. Après avoir appliqué l'optimisation, la température maximale se limite à $270,35{ }^{\circ} \mathrm{C}$ dans les mêmes conditions du fonctionnement. On voit clairement aussi que la différence entre les températures dans les deux cas en fonction de $\mathrm{P}_{\text {diss }}$ est très importante.

\section{Conclusion}

La température du fonctionnement du HEMT influence fortement la performance de ce composant, la plupart de ses caractéristiques ont tendance à se dégrader avec l'augmentation de la température du fonctionnement, notamment la mobilité des électrons. D'autre part, la modélisation thermique par un modèle éléments finis développé sous le logiciel Comsol multiphysics a permis de simuler le comportement thermique du transistor et identifier les paramètres qui ont un grand impact sur ce comportement. Un modèle statistique de l'optimisation par les deux méthodes CMAES et KA-CMAES codées sous le logiciel Matlab, le couplage de ce modèle avec le modèle thermique suivant un organigramme bien précis a permis d'optimiser la température maximale du HEMT, donc améliorer son comportement thermique et sa fiabilité. La méthode KA-CMAES a montré une grande efficacité pour résoudre des problèmes de l'optimisation avec un temps du calcul réduit, mais CMAES est encore couteuse en termes de temps du calcul, d'où l'importance de l'intégration des métamodèles de krigeage dans les problèmes de l'optimisation. Ce travail a pris en considération juste trois paramètres. Pour développer, il est préférable d'introduire autres paramètres et de prendre en considération plus de contraintes afin d'assurer l'efficacité des résultats trouvés.

\section{Bibliographie}

[1] A. El hami, D. Delaux, and H. Grzeskowiak, Reliability of High-Power Mechatronic Systems 1: Aerospace and Automotive Applications: Simulation, Modeling and Optimization. ISTE éditions, 2017.

[2] S. García, I. Ñiguez-De-La-Torre, J. Mateos, T. González, and S. Pérez, "Impact of substrate and thermal boundary resistance on the performance of $\mathrm{AlGaN} / \mathrm{GaN}$ HEMTs analyzed by means of electro-thermal Monte Carlo simulations," Semicond. Sci. Technol., vol. 31, no. 6, 2016, doi: 10.1088/0268-1242/31/6/065005.

[3]L. Baczkowski, "Modélisation et Caractérisation Thermique de Transistors de Puissance Hyperfréquence GaN et Conséquences sur la Fiabilité de Modules Radars d'Émission/Réception en Bande X," Université de Lille, 2015.

[4] Y. Chen et al., "Improved quasi-physical zone division model with analytical electrothermal Ids model for $\mathrm{AlGaN} / \mathrm{GaN}$ heterojunction high electron mobility transistors," Int. J. Numer. Model. Electron. Networks, Devices Fields, no. May, pp. 1-17, 2019, doi: 10.1002/jnm.2630.

[5] Y. Jia, Y. Xu, and Y. Guo, "A Universal Scalable Thermal Resistance Model for Compact Large-Signal Model of AlGaN/GaN HEMTs," IEEE Trans. Microw. Theory Tech., vol. 66, no. 10, pp. 4419-4429, 2018, doi: 10.1109/TMTT.2018.2854185.

[6] S. Samira, "Modeling of Enhancement-Mode GaN-GIT Application," IEEE Trans. Electron Devices, pp. 1-7, 2020.

[7] S. S. Sajjadi Jaghargh and A. A. Orouji, "An AlGaN/GaN HEMT by a reversed pyramidal channel layer: Investigation and fundamental physics," Int. J. Numer. Model. Electron. Networks, Devices Fields, no. December 2019, pp. 13-18, 2020, doi: 10.1002/jnm.2719.

[8] O. Jardel et al., "InAlN/GaN HEMTs based L-band high-power packaged amplifiers," Int. J. Microw. Wirel. Technol., vol. 6, no. 6, pp. 565-572, Dec. 2014, doi: 10.1017/s175907871400004x.

[9] N. Hansen, "The CMA Evolution Strategy: A Tutorial arXiv : 1604 . 00772v1 [ cs . LG ] 4 Apr 2016," 2016.

[10] A. Abdelhamid, H. Hamid, R. Bouchaïb, and E. H. Abdelkhalak, "Optimisation thermique du transistor à haute mobilité d' électron ( HEMT ) par la méthode CMA -ES Thermal optimization of the high electron mobility transistor ( HEMT ) using the CMA-ES method," pp. 10-21, 2020. 
[11] A. Amar, B. Radi, and A. El Hami, "La modélisation thermique de transistor a haute puissance de type HEMT," Incert. fiabilité des systèmes multiphysiques, vol. 3, no. 2019, pp. 1-7, 2019, doi: 10.21494/iste.op.2019.0431.

[12] I. Nifa et al., "Characterization of $2 \mathrm{DEG}$ in AlGaN/GaN heterostructure by Hall effect," Microelectron. Eng., vol. 178, pp. 128-131, 2017, doi: 10.1016/j.mee.2017.05.009.

[13] M. A. Alim, S. Afrin, A. A. Rezazadeh, and C. Gaquiere, "Thermal response and correlation between mobility and kink effect in GaN HEMTs," Microelectron. Eng., vol. 219, no. April 2019, p. 111148, 2020, doi: 10.1016/j.mee.2019.111148.

[14] D. Kara, N. Donmezer, T. F. Canan, O. Sen, and E. Ozbay, "Effects of field plate on the maximum temperature and temperature distribution for gan HEMT devices," Nov. 2016, vol. 1, p. V001T04A003, doi: 10.1115/HT2016-7367.

[15] T.-S. Kang, "Reliability study of algan / gan high electron mobility transistor by thermal simulation and subbandgap optical pumping," University of florida, 2015.

[16] A. J. Tzou, D. H. Hsieh, S. H. Chen, Z. Y. Li, C. Y. Chang, and H. C. Kuo, "Non-thermal alloyed ohmic contact process of GaN-based HEMTs by pulsed laser annealing," Semicond. Sci. Technol., vol. 31, no. 5, p. 0, 2016, doi: 10.1088/0268-1242/31/5/055003.

[17] L. Baczkowski et al., "Temperature measurements in RF operating conditions of AlGaN/GaN HEMTs using IR microscopy and Raman spectroscopy,” Eur. Microw. Week 2015 , vol. 5, pp. 152-155, 2015, doi: 10.1109/EuMIC.2015.7345091.

[18] A. El Hami and P. Pougnet, Embedded Mechatronic Systems 2: Analysis of Failures, Modeling, Simulation and Optimization. ISTE éditions, 2015.

[19] S. Cheng and P. C. Chou, "Novel packaging design for high-power GaN-on-Si high electron mobility transistors (HEMTs),” Int. J. Therm. Sci., vol. 66, pp. 63-70, Apr. 2013, doi: 10.1016/j.ijthermalsci.2012.10.003.

[20] U. Radhakrishna, Physics-based III-Nitride device modeling, 1st ed., vol. 102. Elsevier Inc., 2019.

[21] H. Hamdani, B. Radi, and A. El Hami, "Optimization of solder joints in embedded mechatronic systems via Kriging-assisted CMA-ES algorithm," Int. J. Simul. Multidiscip. Des. Optim., vol. 10, p. A3, 2019, doi: $10.1051 / \mathrm{smdo} / 2019002$.

[22] N. Hansen, N. Hansen, and A. C. M. Nonlinear, "A CMA-ES for Mixed-Integer Nonlinear Optimization To cite this version : HAL Id : inria-00629689 A CMA-ES for Mixed-Integer Nonlinear Optimization,” 2011.

[23] I. Loshchilov, “A Computationally Efficient Limited Memory CMA-ES for Large Scale Optimization,” 2014.

[24] E. Benhamou, J. Atif, R. Laraki, E. Benhamou, J. Atif, and R. Laraki, "A discrete version of CMA-ES To cite this version : HAL Id : hal-02011531 A discrete version of CMA-ES,” 2019.

[25] A. El Hami and B. Radi, Incertitudes, optimisation et fiabilité des structures. Lavoisier, Paris, 2013.

[26] C. Huang, B. Radi, A. El Hami, and H. Bai, "CMA evolution strategy assisted by kriging model and approximate ranking,” Appl. Intell., vol. 48, no. 11, pp. 4288-4304, 2018, doi: 10.1007/s10489-018-1193-3. 